\title{
SHORT COMMUNICATION A Stand-alone Magnetic Bubble Memory
}

\author{
W. KINSNER and B. S. JOLL \\ Department of Electrical Engineering and Industrial Applications of Microelectronics Centre, University of \\ Manitoba, Winnipeg, Manitoba, Canada, R3T 2N2
}

Magnetic bubble memories provide non-volatile, high density, high reliability, low power, solid state data storage. Since the present implementations of bubble memories were expensive, the ultimate goal of bubble technology to provide an alternative to bulky electromechanical disc storage systems could not be realized practically. However, bubble memories might have provided a wide range of capacities for light weight, small-size, low-power, non-volatile storage in systems based on microprocessors. Furthermore, bubble memories could be used as buffers in systems requiring electromechanical disc storage such as floppy discs. Such buffers could substantially extend the operational lifetime of the floppy disc in systems accessing the disc very frequently. A word processor was an example of such a frequent disc-processor transaction system.

This paper described a stand-alone magnetic bubble memory. The memory was independent of any external system architecture. It could provide 92 Kbyte of storage to any system with either asynchronous or synchronous data communication capability. The memory was based on the TIB0203 magnetic bubble module. All the communications and data conversions were carried out by a local MC6802 microprocessor and its associated circuitry. Careful attention had been given to data transfer rates between the stand-alone memory and the digital systems it should serve. Transfer rates greater than that of the TIB0203 were achievable.

This paper also addressed itself to the general interfacing procedures with magnetic bubble memories which were scarcely covered in literature. Specifically, the requirements, architectures and complexities of bubble memory controllers were discussed. 

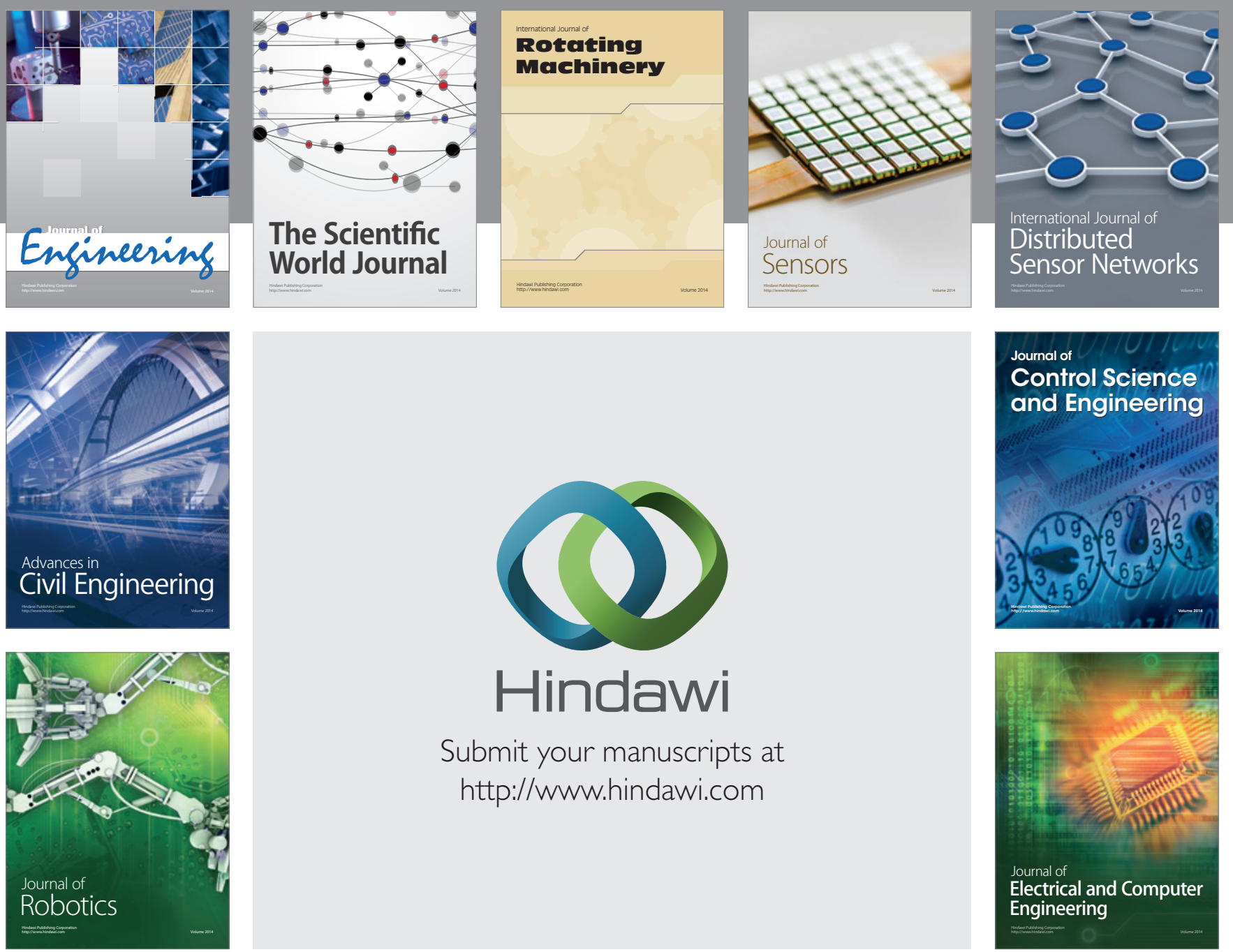

Submit your manuscripts at

http://www.hindawi.com
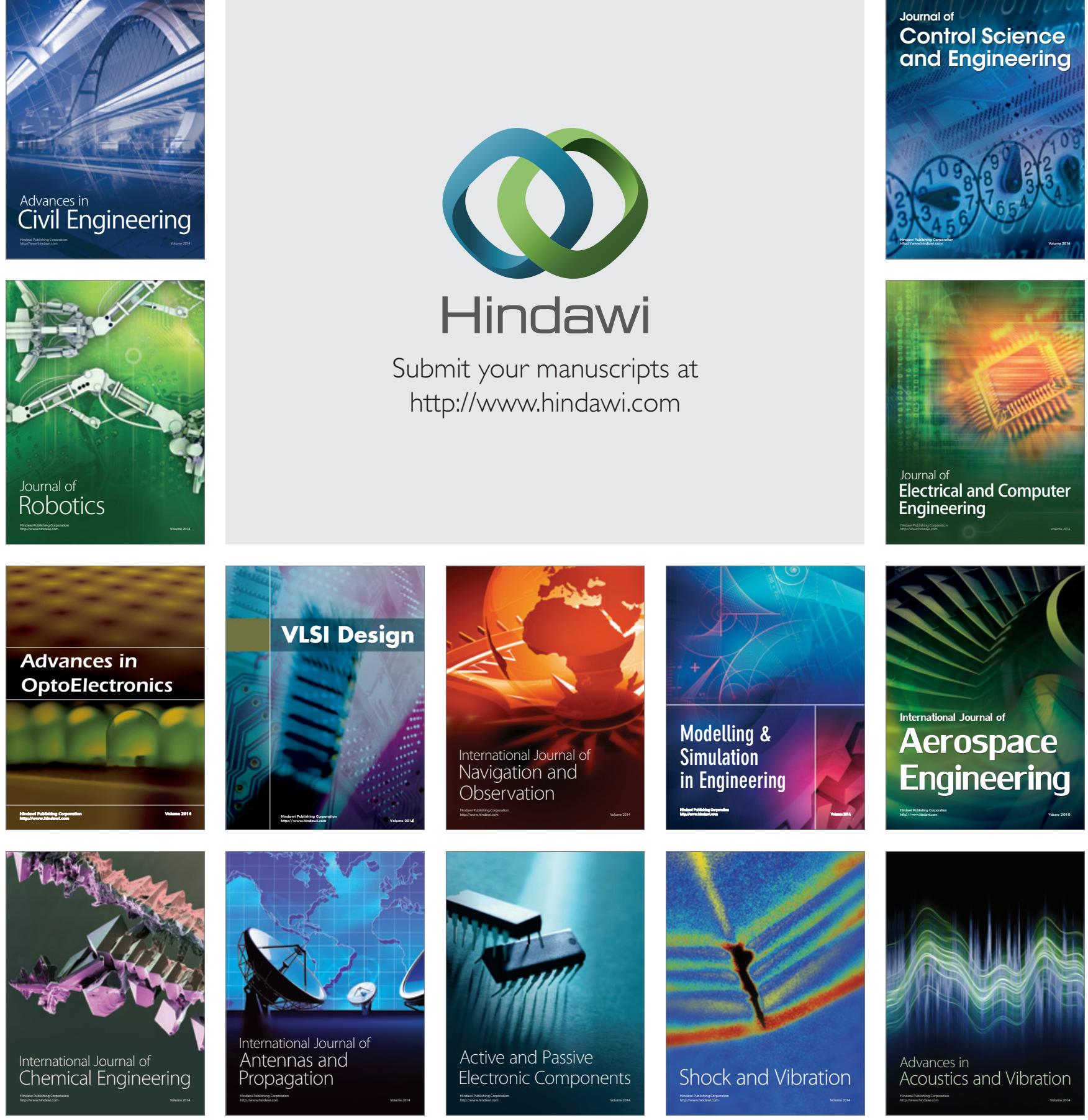